\title{
La gestión del conocimiento para la innovación y el desarrollo rural: experiencias desde Costa Rica
}

\section{Knowledge management for innovation and rural development: experiences from Costa Rica}

\author{
Luis Barboza Arias*, Fernando Sáenz Segura** \\ *Universidad Nacional, Costa Rica. Contacto: luis.barboza.arias@una.cr \\ **Universidad Nacional, Costa Rica. Contacto: fernando.saenz.segura@una.ac.cr.
}

Referencia/ reference:

Barboza, L. y Saénz, F. (2020). La gestión del conocimiento para la innovación y el desarrollo rural: experiencias desde Costa Rica. Yulök Revista de Innovación Académica. 4(1), 21-34

\section{Resumen}

El objetivo del artículo es revisar la evolución conceptual de la gestión del conocimiento, para luego analizar su operacionalización en dos experiencias de extensión en Costa Rica. Se utilizan los estudios de caso como herramienta metodológica y el enfoque de los sistemas de innovación agropecuaria como lente analítico. Se plantea que la gestión del conocimiento rural es un fenómeno relativamente nuevo en Costa Rica. No obstante, las iniciativas presentadas buscan un cambio de paradigma que mejore el abordaje de las problemáticas rurales más complejas. Se concluye con algunas limitaciones del estudio y elementos para avanzar en la construcción de una agenda de investigación en el tema.

Palabras clave: estrategia de desarrollo, planificación de programas, planificación rural.

\section{Abstract}

The objective of the article is to review the conceptual evolution of knowledge management and then analyze its operationalization in two extension experiences in Costa Rica. Case studies are used as a methodological tool and the approach to agricultural innovation systems is used as an analytical lens. It is proposed that rural knowledge management is a relatively new phenomenon in Costa Rica. Nevertheless, the initiatives presented seek a paradigm shift that improves the approach to the most complex rural problems. It concludes with some limitations of the study and elements to advance in the construction of a research agenda on the subject.

Keywords: development strategy, programme planning, rural planning. 


\section{Introducción}

En las últimas décadas un conjunto novedoso de literatura especializada, que se enmarca en el Enfoque de los Sistemas de Innovación Agropecuaria (AIS, por sus siglas en inglés), se ha propuesto analizar los cambios socioproductivos y tecnológicos que han experimentado los territorios rurales desde la revolución verde, para partir de una visión más integral de las dinámicas locales de desarrollo.

Conceptos como gestión del conocimiento y coconstrucción de capacidades, por citar algunos solamente, son utilizados de modo cada vez más frecuente para analizar los factores institucionales que facilitan la conformación de redes para la innovación y estimulan el aprendizaje colectivo en el medio rural.

En Costa Rica, las principales organizaciones públicas del sector agropecuario, entre ellas el Instituto de Desarrollo Rural (INDER), a través de la Unidad especializada de gestión de capacidades y plataformas territoriales, y el Instituto Nacional de Innovación y Transferencia en Tecnología Agropecuaria (INTA), mediante el liderazgo del Foro Relaser-capítulo Costa Rica, han asumido el compromiso de integrar estas nuevas formas de entender las dinámicas rurales a su agenda de trabajo con los actores locales, con el objetivo de consolidar un paradigma de extensión que brinde respuestas efectivas a la creciente complejidad y la interseccionalidad de los desafíos actuales.

De esta manera, iniciativas como el Plan de formación de competencias para los extensionistas, desarrollado por el Foro Relaser-capítulo Costa Rica, surgen con el propósito no solo de modernizar las prácticas de extensión, sino también generar una discusión crítica en torno al tipo de mecanismos que resultan necesarios para mejorar los espacios de coordinación interinstitucional.

Si bien, estas acciones representan un avance importante en materia de fortalecimiento de los instrumentos de apoyo al sector agrícola y productivo nacional, es importante reconocer que aún falta camino por recorrer. La eficiencia y la sostenibilidad de estas propuestas se encuentran condicionadas en gran medida por la adopción oportuna de los arreglos institucionales que permitan la articulación efectiva entre las organizaciones a través de mecanismos claros de gobernanza, toma de decisiones y asignación de recursos y funciones.
El objetivo de este documento es revisar la evolución teórica del concepto de gestión del conocimiento, a partir de la literatura sobre sistemas de innovación agropecuaria, y analizar su operacionalización en el marco de dos experiencias de extensión en Costa Rica.

En la primera de estas se analiza el caso del Foro Relaser-capítulo Costa Rica, que integra a un conjunto diverso de organizaciones públicas en torno a temas y actividades de extensión y trabajo colaborativo con los actores rurales. El segundo caso aborda la experiencia de la Corporación Ganadera de Costa Rica, una institución pública no estatal que en colaboración con la institucionalidad agropecuaria del país y el sector privado implementa, actualmente, el proyecto Piloto Nacional de Ganadería de Carne baja en Emisiones de Gases de Efecto Invernadero, una iniciativa que forma parte del NAMA Ganadería (Acción de Mitigación Nacionalmente Apropiada, que se ejecuta en el país).

Se pretende responder a las preguntas: ¿cómo se articula la gestión del conocimiento a la práctica reciente de la extensión pública y público-privada en Costa Rica? y ¿cuáles han sido los efectos en el desarrollo de territorios rurales?

El documento está organizado de la siguiente manera: El siguiente apartado presenta la metodología utilizada en este estudio. En el tercer apartado se establecen los principales elementos teórico-conceptuales que caracterizan la gestión del conocimiento en el marco del enfoque AIS. El apartado cuatro contiene la discusión de los resultados, a partir de la presentación y discusión de los casos. Finalmente, en el quinto apartado se incluyen las principales conclusiones y algunos elementos para la futura discusión sobre el tema.

\section{Metodología}

En este documento se abordan dos experiencias de extensión pública y público-privada que incorporan la gestión del conocimiento para el desarrollo rural en Costa Rica. Se utiliza el método de estudios de caso en el abordaje y análisis inductivo de la información, que de acuerdo con Yin (1989), facilita el desarrollo de investigaciones empíricas al indagar sobre un fenómeno determinado en su entorno real, a través del uso de múltiples fuentes de evidencia y el establecimiento de patrones de comportamiento, lo que implica un proceso sistematizado de obtención y análisis de datos. 
Como primer estudio de caso se presenta una experiencia de tipo institucional, el Foro Relaser-capítulo Costa Rica, liderada por el Instituto Nacional de Innovación y Transferencia en Tecnología Agropecuaria (INTA). El segundo estudio de caso expone una experiencia de tipo organizacional-productivo, en la que la Corporación Ganadera (CORFOGA), un actor público no estatal, lleva a cabo un proceso de extensión agropecuaria para maximizar las capacidades técnicas de los beneficiarios del Proyecto Piloto Nacional de Ganadería de Carne baja en Emisiones de Gases de Efecto Invernadero, el cual es un instrumento del NAMA Ganadería-Costa Rica.

El abordaje es cualitativo, descriptivo y analítico, con el objetivo de ofrecer una primera revisión del tema de investigación, en función de las preguntas de investigación planteadas. En la recolección de información se hizo uso de una mezcla de fuentes primarias y secundarias. En el primer caso, la recolección se realizó a través de la visita y la consulta a informantes clave de instituciones públicas y corporaciones de productores. Se aplicaron entrevistas semiestructuradas y comunicaciones personales a través de correo electrónico para atender inquietudes puntuales. Se utilizó una guía de entrevista única, en la cual se pidió la opinión de los informantes con respecto a tres variables centrales: 1) grado de conocimiento personal e institucional sobre la gestión del conocimiento; 2) nivel de implementación de la gestión del conocimiento en experiencias de extensión pública abordadas; y, 3) valoración sobre los efectos de la implementación en los territorios rurales. La información secundaria procede en su mayoría de la revisión documental y bibliográfica, en los repositorios de las instituciones públicas relacionadas con esta materia.

\section{Revisión de la literatura y perspectiva analí- tica}

\section{La evolución conceptual de los sistemas de conoci- miento agrícola}

Los sistemas de conocimiento tuvieron su primer desarrollo importante en Nagel (1980), quien planteó el concepto para referirse a la institucionalización de los flujos de conocimiento y el rol de la extensión, luego de observar ambos fenómenos durante su trabajo en dos universidades agrícolas de la India. En estos espacios académicos, el autor abordó la forma en que la investigación, la educación y la extensión eran articuladas por una misma institución (Leeuwis, 2004).
Más adelante, la noción experimentó una modificación importante con la teorización de los Sistemas de Información y Conocimiento Agropecuario (AKIS por sus siglas en inglés), propuesta, originalmente, por los investigadores de la Universidad de Wageningen (Klerkx, Schut, Leeuwis y Kilelu, 2012). Una definición temprana caracteriza el AKIS como un conjunto de organizaciones agropecuarias y/o personas, y los vínculos que tienen lugar a partir de las interacciones entre ellas, con el objetivo de generar, transformar, transmitir, almacenar, recuperar, integrar, difundir y utilizar información, que posibilite los procesos de toma de decisiones y la resolución de problemas asociados con la promoción de innovaciones (Röling, 1989).

Esta forma de entender el AKIS ha sido revisada por diversos autores (Van Woerkum, 1990; Klerkx, Van Mierlo y Leeuwis, 2012; Kilelu, Klerkx y Leeuwis, 2013), quienes argumentan que si bien la interacción en el sistema puede dar paso a nuevas configuraciones y formas de comunicación y colaboración, es precisamente este carácter dinámico del vínculo lo que, también, puede ocasionar la aparición de focos imprevistos de conflicto y escenarios en donde los intereses de los actores pueden llegar a contraponerse.

Una definición posterior por parte de Röling (1992), más receptiva a estas preocupaciones, se refiere al AKIS como un conjunto articulado de actores, redes y organizaciones, que aspiran a trabajar en sinergia, para apoyar los procesos de conocimiento que permitirían un mejor control del entorno a través del uso adecuado de la tecnología. Según esta nueva lectura, el AKIS se convierte en un espacio interactivo, de carácter simbólico, en el que los actores aprenden de manera conjunta, y establecen formas de coordinación y negociación para lograr una adaptación más efectiva al entorno en el que se encuentran (Röling y Wagemakers, 1998).

No obstante, y pese a los cambios de orientación a lo largo del tiempo, es posible distinguir la permanencia de tres componentes fundacionales en la concepción general del enfoque AKIS (figura 1).

En primer lugar, se puede observar el rol protagónico de los actores académicos, principalmente, las universidades, asociados con estructuras verticales de generación de conocimiento sistematizado (Engel, 1995). En segundo lugar, el fuerte énfasis puesto en la disciplina agronómica, como dinamizador de innovaciones técnicas. En este 


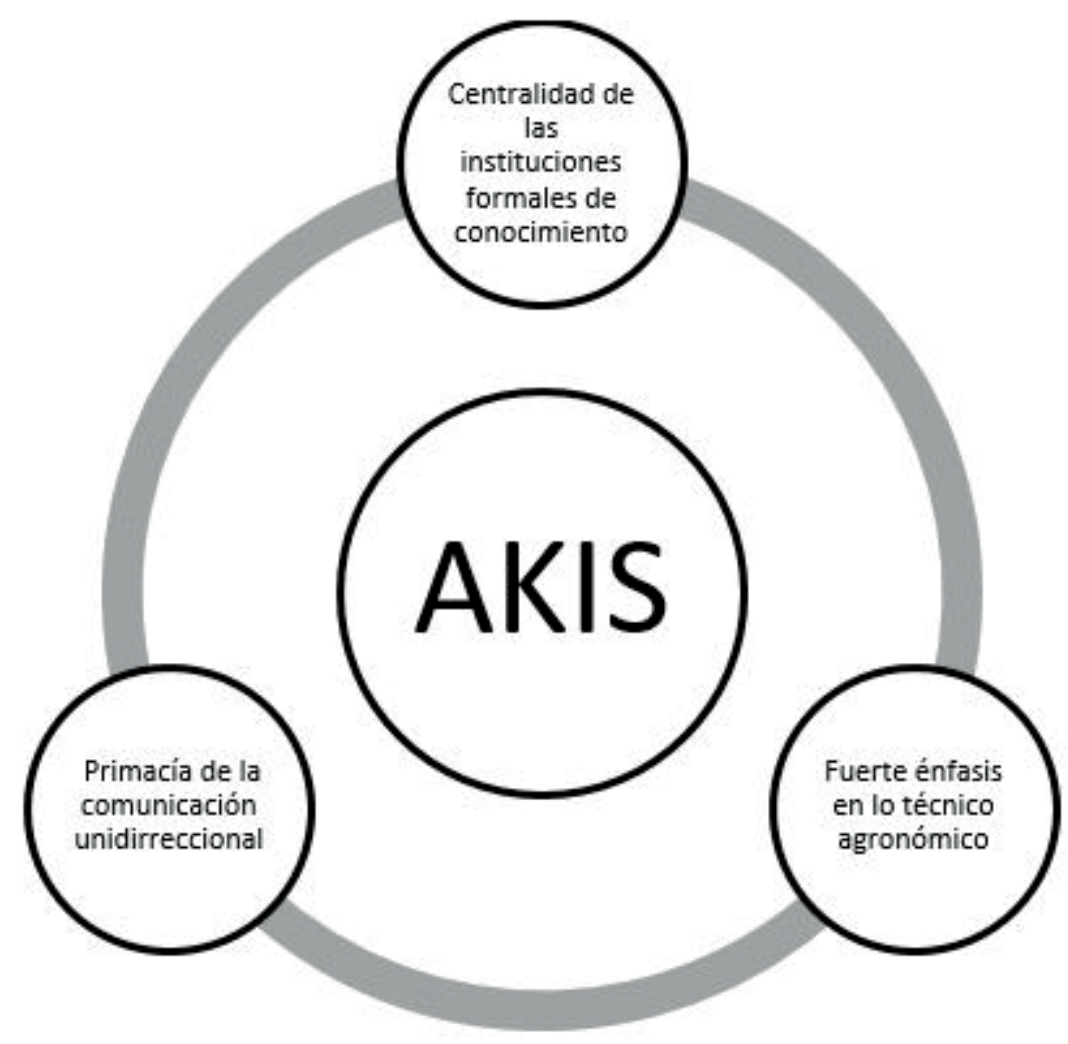

Figura 1. Componentes fundacionales en la concepción general del Enfoque AKIS. Fuente: elaboración propia, 2019.

caso, son los investigadores quienes tienen la atribución y las competencias exclusivas para llevar a cabo los procesos de generación de conocimiento, y se identifica al profesional en Agronomía con la figura de un extensionista experto y calificado (Ingram, 2008). Finalmente, el tercero, donde en el AKIS se atribuye la mayor importancia a los vínculos de comunicación unidireccionales, generalmente, catalogados como procesos de divulgación científica y de información sobre nuevas tecnologías, a través de medios escritos o audiovisuales, que no implican, necesariamente, el contacto directo con otro tipo de actores no académicos, como los grupos de productores, la agroindustria, los consumidores, e incluso, los encargados del diseño de políticas (Klerkx y Leeuwis, 2008; Schut, Rodenburg, Klerkx, van Ast y Bastiaans, 2014).

De forma implícita, esta concepción restringe la aplicación del enfoque al análisis de la dimensión técnica de los procesos de extensión, un aspecto que enfoques complementarios, como el Sistema de Innovación Agropecuaria, han advertido e intentado corregir.

\section{El conocimiento en el marco de los Sistemas de Innovación Agropecuario}

Al igual que sucede en el AKIS, las dinámicas de conocimiento en los Sistemas de innovación agropecuaria (AIS, por sus siglas en inglés) tienen un fuerte carácter institucionalista. El Banco Mundial y la FAO (2000) definen este enfoque como un sistema que integra a un conjunto amplio de actores públicos, privados y de la sociedad civil, y los orienta a la generación de nuevos productos, procesos y formas de organización, con potencial valor económico.

En este sistema, también, toma parte un conjunto de instituciones, entendidas como reglas del juego, y los instrumentos de política (figura 2). Ambos elementos establecen el marco institucional determinado por las interacciones entre los actores y la forma en que estos crean, comparten, acceden, utilizan y se apropian del conocimiento (Yang, Klerkx y Leeuwis, 2014). 


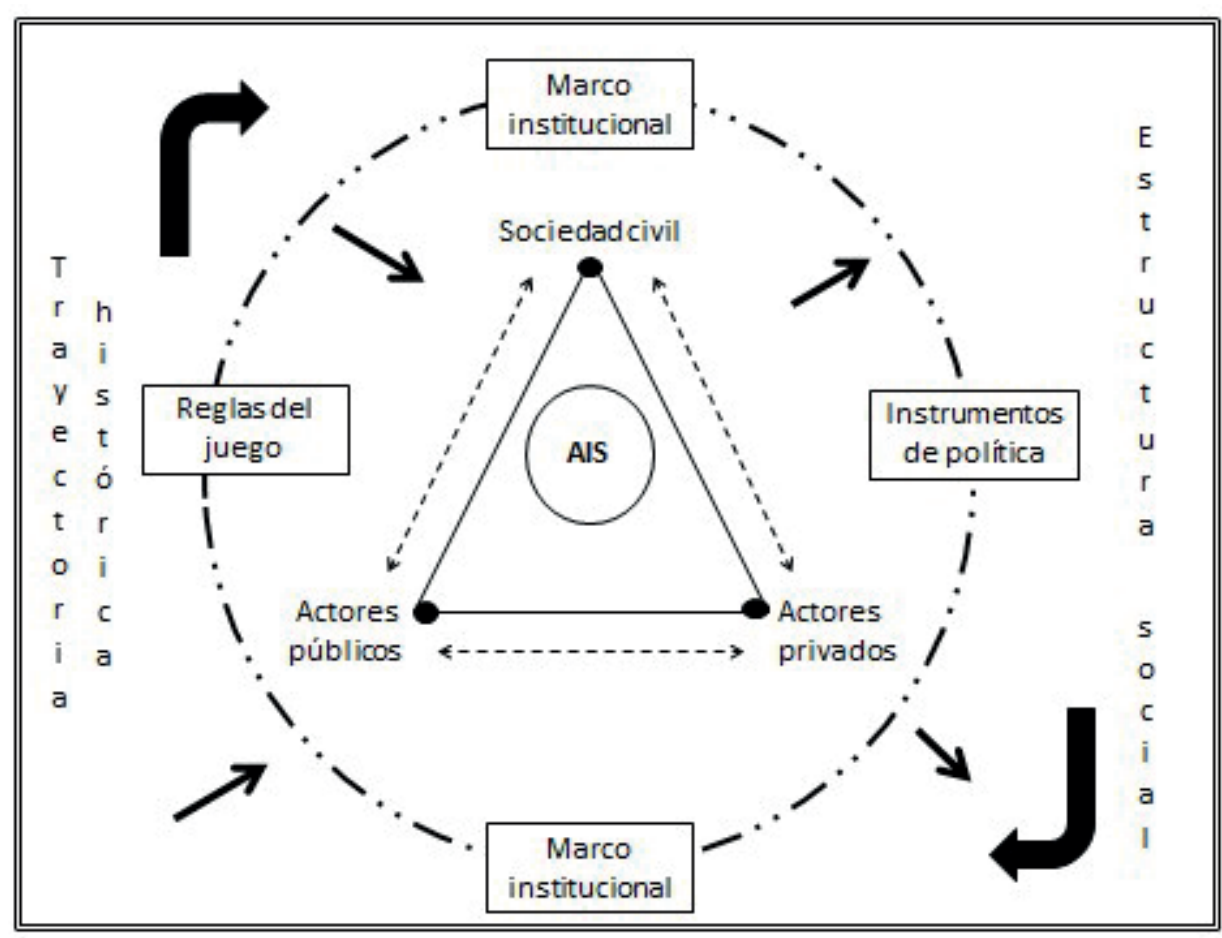

Figura 2. Estructura de los sistemas de innovación agropecuaria. Fuente: elaboración propia, 2019.

Desde la perspectiva del AKIS, los flujos de conocimiento desempeñan una función crucial en la consolidación de modelos de agricultura sostenible, ya que contribuyen a mejorar los niveles de eficiencia, productividad y competitividad de las unidades productivas, a través del uso adecuado de las tecnologías disponibles (Aguilar-Gallegos, Muñoz-Rodríguez, Santoyo-Cortés, Aguilar-Ávila y Klerkx, 2015).

Sin embargo, el enfoque reconoce que una interacción débil entre los actores puede provocar bajos niveles de adopción tecnológica y afectar la calidad de los vínculos. Esta situación se presenta cuando los aspectos socioculturales y ambientales dejan de ser considerados como parte de las fuerzas motrices que configuran las dinámicas históricas del entorno (Dogliotti et al., 2014).

Para evitar que aparezcan estas fallas en la estructura del sistema, autores como Klerkx y Jansen (2010) y Dentoni y Klerkx (2015) han señalado la necesidad de incorporar a la discusión pública sobre los sistemas nacionales de innovación agropecuaria, el análisis crítico de la complejidad de los sistemas sociales y la diversidad de formas de organización que se encuentran en el nivel local, con el objetivo de mejorar la racionalidad y el alcance de los esquemas de extensión.
Dicho de otro modo, no es posible obviar que las actividades de investigación e innovación están insertas en un contexto más amplio de interacción humana. Por lo tanto, su relevancia para la sociedad no depende, únicamente, de las aspiraciones profesionales de los investigadores, sino más bien de su capacidad reflexiva para coadyuvar a superar los vacíos de conocimiento a través de una gestión integral de las dinámicas rurales (Darnhofer, Schermer y Schneeberger, 2008; Hermans, Klerkx y Roep, 2015).

\section{La gestión del conocimiento como creación de capacidades locales}

Diversos autores se han mostrado críticos al señalar que, si bien el futuro de la agricultura y los sistemas agroalimentarios podría depender de la modernización tecnológica, a través del uso de la biotecnología y de la robótica agrícola (Petit, 2015; Godin y Vinck, 2017), los resultados de la progresiva mecanización del medio rural pueden conducir a una mayor exclusión social, la reducción de empleos y la desarticulación de las bases productivas en el nivel local (Duckett, Pearson, Blackmore y Grieve, 2018; Temple y Sawadogo, 2018).

Aunado a esto, tampoco es posible obviar que la agricultura es uno de los sectores más vulnerables a los efectos del cambio climático (Touzard, 2018), por lo que resulta 
necesario identificar y adoptar nuevos paradigmas que permitan el abordaje de estos escenarios en un marco de acción integral (Fabrivious y Cundill, 2014).

Pese a que el reto es claro, las soluciones no son tan sencillas. Por lo que en la literatura reciente se ha comenzado a discutir con mayor detenimiento sobre los procesos de gestión y coconstrucción de capacidades (Turner et al., 2017; Šūmane et al., 2018). En estos estudios, se parte del análisis de la dimensión socio-organizacional que acompaña el diseño de las políticas y esquemas de innovación y extensión, en un esfuerzo por superar el reduccionismo tecnocientificista que primó en las décadas anteriores (Leeuwis, 2004).

Asimismo, se incorpora una perspectiva de gobernanza multinivel para evidenciar la importancia que tiene la participación de los actores sociales en la construcción de redes locales de conocimiento (Siebenhüner, Rodela y Ecker, 2016). Esta nueva narrativa apela al cambio institucional, como un punto de partida para el establecimiento de espacios horizontales de negociación y de búsqueda de acuerdos (Bennett et al., 2015; Meléndez y Parker, 2018). Para Wigboldus et al. (2016), este es uno de los puntos más sensibles, ya que los mecanismos tradicionales de gestión del aprendizaje no incorporan el abordaje de la tensión y el conflicto como parte de sus componentes operativos.

La coconstrucción de conocimiento, por lo tanto, implica mucho más que la puesta en práctica de dispositivos institucionales que estimulen el aprendizaje colectivo y la absorción de las tecnológicas, según el modelo de agricultura vigente (Arkesteijn, van Mierlo y Leeuwis, 2015). De acuerdo con Suškevičs, Hahn, Rodela, Macura y Pahl-Wostl (2018), coconstruir conocimiento significa identificar una ruta de transición hacia un futuro sostenible, en la que se considere lo siguiente:

- La incorporación de cambios sustantivos en la forma de abordar las necesidades, problemáticas y expectativas de los actores locales, incluyendo a aquellos cuyas actividades productivas no son intensivas en el uso de tecnologías, pero que tienen un efecto directo en el sistema socioecológico (Siebenhüner et al., 2016; Gerlak et al., 2018).

- La integración armoniosa de las prácticas locales y las instituciones informales, a través de procesos colaborativos y acciones colectivas destinadas a generar tejido social y organizacional en el medio rural (Suškevičs et al., 2018),
- La generación de un nuevo tipo de pedagogía del cambio (Frisk y Larson, 2011), que trascienda los espacios educativos formales y se inserte en la vivencia cotidiana local, a través de nuevas lógicas de coordinación, medición y evaluación del trabajo con los actores locales (Schmid, Knierim y Knuth, 2016).

\section{Resultados}

\section{La gestión del conocimiento en el marco de las políticas agropecuarias y de desarrollo ru- ral de Costa Rica}

La gestión del conocimiento es un tema de discusión reciente en las políticas dirigidas al desarrollo agropecuario y rural en Costa Rica. El término adquirió importancia en el contexto de los procesos de modernización de los servicios de innovación y extensión agropecuaria promovidos por la institucionalidad pública desde inicios de la primera década del presente siglo.

En el 2001 se creó por medio de la Ley 8149 el Instituto Nacional de Innovación y Transferencia en Tecnología Agropecuaria (INTA), un órgano de desconcentración máxima adscrito al Ministerio de Agricultura y Ganadería (MAG), cuya misión es contribuir con el sector, al disponer de opciones, servicios y productos tecnológicos que son resultado de su gestión en investigación, innovación y transferencia de tecnología. En el 2004, el INTA creó con el apoyo de la Organización de las Naciones Unidas para la Alimentación y la Agricultura (FAO), la Plataforma de Tecnología, Información y Comunicación Agropecuaria y Rural (PLATICAR). Esta plataforma virtual busca mejorar la gestión del conocimiento a través de procesos de mediación pedagógica en línea.

En el 2012, por medio de la Ley 9036, se produjo la transformación del Instituto de Desarrollo Agrario (IDA) en el Instituto de Desarrollo Rural (INDER). Con la Ley 9036 se creó, además, el Fondo de Desarrollo Rural, que tiene como uno de sus principales objetivos, gestionar el acceso por parte de los productores rurales a los conocimientos, la información, el desarrollo tecnológico y los servicios de apoyo requeridos para innovar productos y procesos. Al igual que el INTA, el INDER está agrupado en torno a la figura del MAG, ente rector en materia de agricultura y ganadería.

La gestión del conocimiento agropecuario, también, ha formado parte de las propuestas de gobierno presentadas por las administraciones recientes. La Política de Esta- 
do para el sector agroalimentario y el desarrollo rural costarricense 2010-2021 (SEPSA, 2011), que deriva de las prioridades definidas para el sector agropecuario en el Plan de Gobierno 2010-2014, de la Administración Chinchilla Miranda, no solo identificó este tema como una de las áreas estratégicas para la competitividad y el desarrollo tecnológico, además propuso una serie de mecanismos para garantizar una adecuada gobernanza y la inclusión de los pequeños y medianos productores. Esta Política estableció un marco regulatorio para la gestión del conocimiento y sus respectivas instancias de coordinación, y promovió la ejecución de instrumentos específicos para disminuir las brechas de acceso a la información y el conocimiento. No obstante, estas iniciativas no parecen haber alcanzado los resultados previstos. Si bien existía claridad con respecto al tipo de enfoque que debía implementarse en el momento de su diseño, no se han destinado los recursos institucionales y económicos suficientes para definir instrumentos de política impulsados por la demanda, que, además, se encuentren en sintonía con la infraestructura local y las capacidades reales de los productores.

Las Políticas para el sector agropecuario y el desarrollo de los territorios rurales 2015-2018, promovidas por la Administración Solís Rivera (2014-2015), fueron en cambio más modestas. Como áreas estratégicas para la gestión del conocimiento se definieron el establecimiento de alianzas con la academia para el intercambio de información y la capacitación de productores y técnicos, el establecimiento de mecanismos de información, verificación y control, la coordinación interinstitucional para apoyar a grupos sociales específicos y la utilización de las fincas integrales didácticas y vitrinas tecnológicas como centros de construcción colectiva del conocimiento (SEPSA, 2014). Estas acciones se articularon de forma transversal a dos temas complejos, que fueron prioritarios en la agenda de desarrollo de la Administración Solís Rivera: la seguridad alimentaria y nutricional y la adaptación al cambio climático en los territorios rurales.

De forma reciente, los Lineamientos de política 2019-2022 para el sector agropecuario, pesquero y rural, presentados por la Administración Quesada Alvarado (2018-2012), mantienen la línea de la Administración Solís Rivera en materia de gestión del conocimiento y fortalecimiento de capacidades técnicas en cambio climático y gestión del riesgo (SEPSA, 2018). No obstante, la agenda del actual Gobierno considera crucial centrar las iniciativas de gestión del conocimiento en los grupos sociales más vulnerables, mujeres y juventud rural en particular, con el objetivo de favorecer dinámicas de generación de

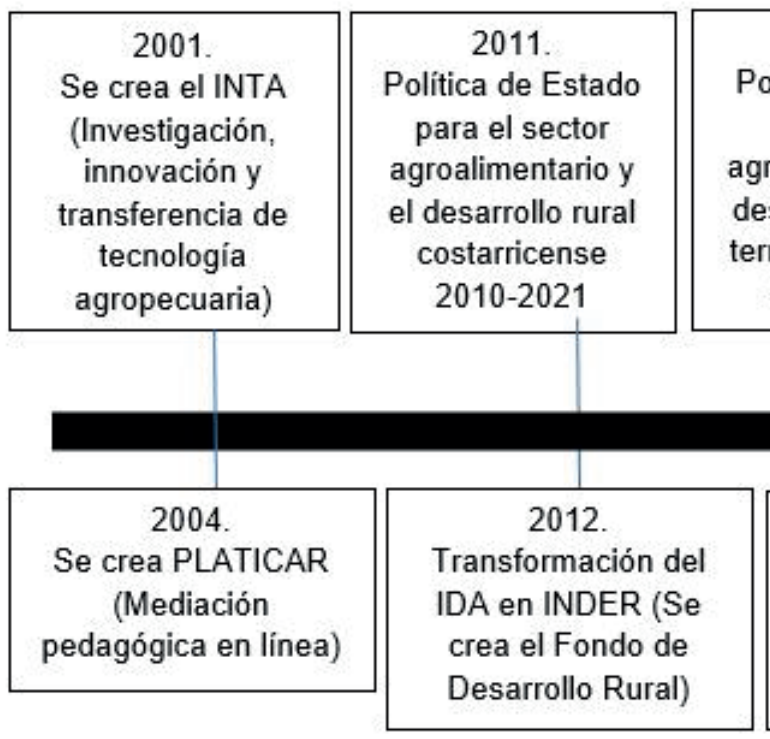

\section{4.}

Políticas para el sector agropecuario y el desarrollo de los territorios rurales 2015-2018

Figura 3. Evolución histórica de los principales instrumentos de política en Costa Rica que incluyen la gestión del conocimiento agropecuario. Fuente: elaboración propia, 2019.

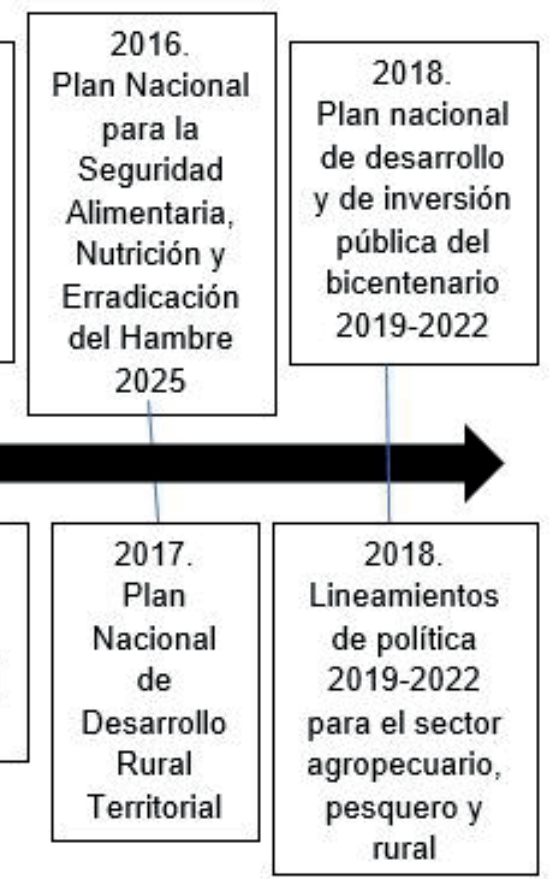


empleo, agregación de valor y mayor equidad social. A manera de ejemplo, en el 2018, la pobreza extrema en la Región Central de Costa Rica, considerada la zona más urbana del país, fue de $4,4 \%$, mientras que, en regiones rurales como la Región Brunca y la Huetar Norte, fue de $10,1 \%$ y $10,0 \%$, respectivamente. Con respecto al desempleo, la población rural más afectada por el desempleo sigue siendo los jóvenes entre 15 y 34 años (INEC, 2019). En la figura 3 se presenta una línea de tiempo con los principales instrumentos de políticas que incluyen la gestión del conocimiento agropecuario. (figura 3 )

\section{El nuevo rol del conocimiento en la exten- sión agropecuaria y rural: el Foro Relaser-ca- pítulo Costa Rica}

La Red Latinoamericana para Servicios de Extensión Rural (RELASER), creada en 2010, es una red regional compuesta por un colectivo diverso de instituciones y profesionales vinculados a la extensión rural en América Latina, actualmente, participan en ella nueve países, a los cuales se les denomina Foros Nacionales. Su misión es apoyar la consolidación de los sistemas de extensión públicos y privados en la región, a través de la promoción del desarrollo sostenible y los sistemas de innovación. RELASER forma parte del Foro Global para los Servicios de Asesoría Rural (GFRAS), un organismo con presencia internacional que brinda apoyo a organizaciones públicas y grupos de productores en diferentes partes del mundo, con el objetivo de mejorar la prestación de servicios de extensión rural y las relaciones de las instituciones con los actores locales.

En su esquema operativo, estos organismos incorporan una visión heterodoxa de conceptos como acción colectiva y aprendizaje social, con la finalidad de ampliar el marco analítico y comprender de mejor manera la complejidad y multisectorialidad del desarrollo rural (Therond, Duru, Roger-Estrade y Richard, 2017).

En enero del 2014, el Foro Relaser-capítulo Costa Rica se conformó como un espacio de intercambio y articulación interinstitucional, cuyo principal objetivo es la incidencia de políticas, a través del replanteamiento del esquema de extensión agropecuaria, hasta ese momento, vigente. Actualmente, lo integran representantes de instituciones públicas (Dirección de Extensión Agropecuaria (del MAG, INTA, INDER y Movimiento de Clubes 4S), actores académicos (Universidad de Costa Rica, Universidad Estatal a Distancia, Universidad Técnica Nacional), instituciones privadas y organismos internacionales, entre ellas la FAO.

La experiencia costarricense del Foro Relaser representa un esfuerzo importante por superar la fragmentación institucional y reorientar las funciones de cada ente hacia un esquema colaborativo y el mejoramiento articulado. La participación de este conjunto diverso de actores ha permitido en el nivel programático la generación de información valiosa sobre el estado de la extensión en el país y cómo potenciarla a través de enfoques innovadores, que promuevan la movilización de una mayor cantidad de actores de la sociedad civil hacia la transformación productiva.

El interés creciente por la consolidación de mecanismos de participación de las fuerzas sociales en el proceso de cambio y mejora de la extensión favoreció el establecimiento de un debate a lo interno del Foro Nacional, sobre el tipo de instrumentos que son necesarios para responder a las especificidades y problemáticas de los diferentes grupos sociales y productivos en cada territorio rural. Como resultado de esas reflexiones, las organizaciones que conforman el Foro han comprendido la importancia de entender la gestión del conocimiento como un enfoque dinámico y evolutivo, necesario en cualquier estrategia de coordinación, cooperación y comunicación.

La discusión interinstitucional dio paso a una revisión de las capacidades estatales, a partir de lo cual ha sido posible la introducción paulatina de cambios institucionales. También, se definieron cuatro temas prioritarios para su abordaje por parte del Foro, entre ellos, el desarrollo de capacidades, habilidades y destrezas en extensionistas, la articulación de los servicios de extensión con el sistema de innovación, los espacios de diálogo y gestión de conocimiento y el seguimiento y evaluación del impacto de la extensión.

Uno de los cambios institucionales más importantes es la incorporación de la gestión del conocimiento como instrumento sistemático en el desarrollo y fortalecimiento de las capacidades y competencias no solo de productores, sino también de los extensionistas. De este modo, el Plan de Formación de Competencias Complementarias para los Extensionistas (INTA, 2018) se ha convertido en un insumo importante en la práctica de la extensión agropecuaria, precisamente, porque plantea un cambio significativo en la perspectiva institucional de lo rural, al abrir el enfoque de los aprendizajes centrados en la formación 
técnica a un conjunto más amplio de procesos, como la formación de redes y la promoción de acciones colectivas que fortalezcan el tejido social en las organizaciones y comunidades.

Este último aspecto es importante, ya que favorece la visibilización de una agenda rural que hasta entonces no había sido planteada de forma explícita en el ámbito público, y que se relaciona con la necesidad de integrar los esquemas de extensión, transferencia tecnológica y capacitación a una política de desarrollo territorial rural basada en la promoción del rol de la mujer y la juventud rurales, y que tiene un efecto importante en términos de la discusión sobre estrategias para garantizar el relevo generacional y la equidad de género en la agricultura y el medio rural.

Este mismo razonamiento está presente en otros documentos elaborados por el Foro Relaser-capítulo Costa Rica, entre ellos, el Manual de buenas prácticas de extensión rural: caso Costa Rica (2016) y el Informe sobre competencias del extensionista de cara al relevo generacional en Costa Rica (2017), en cuyos planteamientos es posible identificar la adopción de los nuevos paradigmas a los que se refieren autores como Fabrivious y Cundill (2014) y Suškevičs (2018).

\section{El fomento de capacidades técnicas y so- cioproductivas en un contexto de transición sostenible: la experiencia de la Corporación Ganadera}

La Corporación Ganadera (Corfoga), de acuerdo con la Ley 7837, que establece su creación, es una institución de derecho público, no estatal, cuyo objetivo principal es el fomento de la ganadería bovina de Costa Rica, a través de la promoción y apoyo a la transformación tecnológica y empresarial de la ganadería y los segmentos de la cadena agroindustrial de la carne.

En términos de organización, el sector cárnico bovino de Costa Rica está conformado por 28 cámaras/asociaciones de ganaderos, que se encuentran integradas a su vez en tres federaciones con presencia territorial, las cuales son encargadas de servir como el medio de enlace entre los productores y la coordinación sectorial a cargo de Corfoga.

El Proyecto Piloto Nacional de Ganadería de Carne baja en Emisiones de Gases de Efecto Invernadero es una iniciativa institucional impulsada por Corfoga en el año 2013 con el objetivo de implementar un modelo piloto de gestión de desarrollo ganadero a nivel nacional por medio de estructuras regionales que permita el fortalecimiento de la cadena cárnica. El Proyecto Piloto estaba integrado, originalmente, por cuatro componentes: productivo, de extensión, financiero y comercialización, y se orientaba a la mejora de aspectos de productividad y rentabilidad de los ganaderos inscritos en las diferentes cámaras y asociaciones.

No obstante, en el año 2015, con el inicio de la implementación del NAMA Ganadería de Costa Rica, un instrumento de política pública para la reducción de emisiones de gases de efecto invernadero, gestionado por el Ministerio de Agricultura y Ganadería (MAG), el Proyecto Piloto se convierte en uno de sus principales instrumentos operativos y se le incorpora la variable ambiental. Desde entonces, a través del NAMA se ha venido trabajando en el desarrollo de prácticas de mitigación y adaptación que, inicialmente, no estaban consideradas dentro del Proyecto Piloto.

Esta evolución de instrumentos dio paso a un arreglo institucional que favoreció la identificación de compromisos y responsabilidades por parte de diferentes actores institucionales, encabezados por el MAG, el Ministerio de Ambiente, Energía y Telecomunicaciones (MINAET), y el sector ganadero. En este sentido, el diseño de la Estrategia de Ganadería Baja en Carbono (EGBC) surgió de manera posterior como un mecanismo interinstitucional de carácter integral que se propone articular las acciones en materia ambiental y socioproductiva implementadas a nivel sectorial por el NAMA Ganadería a una agenda de cambio climático de alcance local y nacional.

En este contexto, el rol de Corfoga como interlocutor del sector privado ha sido crucial. El Proyecto Piloto, como ejercicio práctico de implementación del NAMA Ganadería, ha dado paso a un proceso de aprendizaje institucional importante. El análisis técnico asociado al diseño de métricas, y la medición en sí misma, como es el caso de la técnica MRV (Medición, Reporte y Verificación, por sus siglas en inglés), la cual manifiesta la necesidad de generar los mecanismos adecuados para estimular y fortalecer las capacidades institucionales con respecto a la investigación científica en materia ambiental (el diseño de coeficientes, por ejemplo), las estructuras de extensión y formación técnica de los Ministerios, y la transferencia de tecnologías al ganadero. 
Precisamente, uno de los principales resultados obtenidos con el Proyecto Piloto Nacional de Ganadería de Carne baja en Emisiones de Gases de Efecto Invernadero ha sido la validación de las metodologías y procesos utilizados en la selección e implementación de tecnologías específicas y el acompañamiento al productor. De igual manera, la generación de evidencia empírica e información oportuna sobre los efectos de estas tecnologías en el ambiente y el desempeño de la actividad productiva solo ha sido posible por la integración efectiva de los ganaderos, quienes participan, activamente, en talleres y giras demostrativas, en donde exponen sus experiencias a partir de los cambios introducidos y las nuevas prácticas de campo, así como las barreras de implementación encontradas, a través de metodologías participativas de gestión del aprendizaje.

Las escuelas de campo se han convertido en un elemento innovador en la práctica de extensión realizada por Corfoga. En el marco del Proyecto Piloto, son los ganaderos quienes se convierten en capacitadores de otros ganaderos. Mediante el intercambio horizontal e interactivo de conocimiento, los ganaderos comparten las prácticas que les han resultado beneficiosas y las oportunidades de mejora, dadas las condiciones particulares del sistema socioecológico de su finca y entorno. Estas prácticas, que podrían denominarse de coconstrucción de conocimiento permiten generar una dinámica de aprendizaje social y colectivo que trasciende la dimensión técnica y permite incorporar la dimensión simbólica y subjetiva, a través de las aspiraciones y expectativas de los ganaderos.

En su primera fase, el Proyecto Piloto incluyó a 100 fincas de productores de todo el territorio nacional. Estas fincas, a las cuales se les ha llamado fincas experimentales, constituyen laboratorios vivos no solo por su contribución al desarrollo de una ganadería climáticamente inteligente, sino también por su efecto en la creación de capacidades locales. De esta manera, además del desarrollo de actividades para la mejora de la administración de la pastura y la incorporación de árboles y arbustos en las fincas ganaderas, el Proyecto Piloto, también, promueve la organización sectorial y la creación de redes mediante el diálogo de saberes, en donde son los actores locales (los ganaderos, en este caso) los protagonistas. Estos aspectos han sido discutidos en detalle por Bennett et al. (2015) y Siebenhüner et al. (2016), quienes incorporan la dimensión de los liderazgos colaborativos como una variable destacada en la articulación de políticas sectoriales y políticas de desarrollo.
En el 2019, el Proyecto Piloto avanza hacia su quinto año de implementación y una segunda etapa, y se propone continuar fortaleciendo los mecanismos de extensión, de manera que se garantice que los enfoques implementados permitan una adecuada integración de los productores. En este sentido, se han hecho esfuerzos importantes para legitimar, en el marco de la planificación institucional, elementos del conocimiento tácito y aplicado.

En esta nueva etapa, el Proyecto Piloto continuará colaborando con 25 fincas de las cien que, originalmente, integraron la primera etapa, en actividades de difusión y transmisión de conocimiento con el esquema de productor a productor. No obstante, no existe un mecanismo identificado para dar seguimiento a los ganaderos que no continúan, lo cual puede entenderse como una debilidad importante.

En términos de desarrollo inclusivo, resulta igualmente importante considerar que la continuidad de la colaboración con las fincas de ganaderos que han logrado resultados notables puede conducir al debilitamiento de los resultados menores que lograron las fincas que no se mantienen en la segunda etapa. En este sentido, si bien las fincas que continúan se convertirán en fincas escuelas, tampoco debe obviarse que existen barreras estructurales no solo en términos de conocimiento o recurso tecnológico, sino también con respecto al financiamiento y la fluidez económica para invertir. Estos son temas que afectan a los pequeños y medianos productores.

Dicho de otra manera, pese a que a nivel técnico es posible identificar progresos importantes en las áreas de mejoramiento genético, técnicas de pastoreo, ensilado y rastreabilidad, entre otras, no basta con lograr un cambio de actitud en el productor. Los instrumentos como el Plan Piloto, por ende, deben servir también como base empírica para propiciar una mayor discusión intersectorial sobre la importancia del uso responsable de los recursos locales y las políticas de bajo costo en escenarios de restricción presupuestaria.

\section{Discusión}

En términos de las preguntas de investigación a las que este artículo se propone contribuir, cabe mencionar que la incorporación de la gestión del conocimiento a la práctica de la extensión pública y público-privada en Costa Rica es un fenómeno relativamente nuevo. 
Como resultado del análisis de la evolución histórica de los principales instrumentos de política agropecuaria y para la ruralidad, es posible evidenciar que, pese a que existe un interés institucional en generar una discusión en torno al tema, en materia de operacionalización del concepto a través de actividades de extensión específicas, aún no se dispone de una estrategia de trabajo articulada.

En este sentido, las experiencias como el Foro Relaser-capítulo Costa Rica representan una ruptura de paradigma que contribuyen a generar el debate en torno a la pertinencia de ampliar el análisis de los procesos de transferencia de tecnologías y asesoría técnica al estudio de variables que no corresponden de forma precisa al ámbito técnico de la innovación y la modernización del medio rural.

El hecho de pensar la innovación en relación con las dinámicas rurales más complejas, y no solamente en función de su contribución al desarrollo agroproductivo, permite entender en qué medida los aspectos socioculturales y ambientales que están transformando a la ruralidad costarricense tienen un efecto directo en el modo de concebir e implementar los enfoques de extensión más recientes.

Autores como Dentoni y Klerkx (2015) y Dogliotti et al. (2014) habían problematizado la influencia de estos determinantes del entorno en el diseño de instrumentos y políticas, al referirse a la atención creciente que brinda el enfoque de los sistemas nacionales de innovación al estudio de las interacciones entre los actores en diferentes niveles del sistema.

De forma implícita, existe el reconocimiento por parte de la institucionalidad agropecuaria, tanto pública como privada, de que las organizaciones formales no son los únicos actores competentes para generar procesos de esta naturaleza. En los estudios de caso abordados se destaca la discusión previa entre actores que condujo al establecimiento de arreglos institucionales básicos, con el objetivo de incorporar una perspectiva dinámica y reflexiva que permitiera la apertura de espacios horizontales y de diálogo con actores locales.

Si se analiza esta dimensión desde una lógica de mercado, lo anterior equivale a decir que ninguna estrategia de gestión del aprendizaje rural puede ser exitosa si no se toman en cuenta las demandas reales y las competencias existentes entre los colectivos y territorios específicos a los cuales esta clase de iniciativas están dirigidas. Conviene, por lo tanto, avanzar en una agenda integral que permita caracterizar los perfiles de los diferentes tipos de actores rurales. En la reflexión teórica, este tema ha sido conceptualizado por Suškevičs et al. (2018). Estos autores plantean que el reconocimiento de las acciones y de base son un punto de partida, e incluso un requisito previo, para la creación de redes y estructuras de apoyo que garanticen una adecuada gestión del conocimiento.

Con respecto a dinámicas sectoriales, como es el caso del Proyecto Piloto Nacional de Ganadería de Carne baja en Emisiones de Gases de Efecto Invernadero, resulta valioso destacar que los cambios en la forma de abordar las problemáticas y expectativas de los ganaderos que participan en esta iniciativa condujeron a Corfoga a un proceso de reflexión crítica. En este sentido, el Proyecto piloto se ha convertido de forma reciente en un instrumento de validación con los ganaderos, y constituye un medio interactivo para la reproducción de acciones coordinadas que incorporan elementos sociológicos y subjetivos, tales como identidad y confianza. Desde un punto de vista teórico, Leeuwis, (2004) llama a esto el enfoque interactivo para la comunicación y la innovación rural.

En Siebenhüner et al. (2016) y Gerlak et al. (2018), también, se encuentran elementos teóricos para entender esta clase de cambios en el enfoque analítico de la gestión del conocimiento. En sus estudios, se concluye con la idea del cambio sustantivo y la necesidad de avanzar en la configuración de nuevas formas de relacionamiento entre personas, dispositivos técnicos y fenómenos naturales.

\section{Conclusiones}

Como se desprende de los estudios de caso abordados en esta investigación, una de las principales limitaciones es la falta de metodologías de evaluación focalizadas en procesos de gestión del conocimiento. En consecuencia, las actividades de extensión que incorporan dinámicas innovadoras en esta materia continúan siendo evaluadas mediante el lente de la extensión tradicional. Esta situación no permite observar procesos como la influencia de las interacciones en el aprendizaje o los efectos cognitivos que tiene en el productor la validación institucional del conocimiento tácito y su consecuente integración a esquemas de capacitación basados en el conocimiento sistematizado y explícito.

Si bien las iniciativas presentadas en este artículo son conscientes de las barreras estructurales que enfrentan 
los actores rurales, la agenda de promoción de la gestión del conocimiento en ambos casos carece de una perspectiva intersectorial que le permita dialogar con otras dimensiones inherentes al desarrollo rural, por ejemplo, la disponibilidad y calidad de los servicios educativos, sanitarios y de generación de empleo en los territorios rurales. La ausencia de indicadores y metodologías adecuadas para evaluar de forma efectiva la funcionalidad y pertinencia de los instrumentos implementados en las experiencias citadas representa un vacío conceptual que requiere necesariamente un abordaje sistemático posterior.

No obstante, en términos analíticos es posible indicar que la gestión del conocimiento que se presenta en los estudios de caso ha contribuido a mejorar la construcción de vínculos y redes de apoyo entre la institucionalidad pública y privada del sector agropecuario, de tal manera que se ha incluido un incremento en la participación de los productores en procesos de toma de decisiones y diseño de instrumentos.

La incorporación de la gestión del conocimiento condujo a nuevas formas de relacionamiento social entre actores institucionales y productivos, que resultan claves para poner en práctica los mecanismos de apropiación del conocimiento, como es el caso del esquema de productor a productor y las fincas escuelas que promoverá Corfoga en la segunda etapa del Proyecto piloto.

Resulta claro que pese a la gestión del conocimiento en Costa Rica avanza hacia un esquema basado en la horizontalidad y el empoderamiento de los actores agroproductivos locales. En la práctica, algunos de estos mecanismos son racionalizados con base en una perspectiva normativista, que continúa otorgándole a las instituciones públicas el rol de entes de articulación en cualquier iniciativa de cambio. Esta narrativa tradicional, sin duda alguna, lleva implícita la marca de las políticas de arriba hacia abajo que discuten Schmid et al. (2016) y Hermans et al. (2015).

El trabajo aquí presentado proporciona una valiosa información sobre los avances y cambios cualitativos en la gestión del conocimiento en la práctica de la extensión en Costa Rica. No obstante, es importante reconocer que existen desafíos operativos que deben ser atendidos si el propósito que se plantea es convertir a la gestión del conocimiento en un instrumento efectivo de desarrollo rural. Entre estos desafíos se encuentran la necesidad de incorporar una perspectiva multinivel y multiactor que conduzca a un fortalecimiento de la gobernanza en este sistema. También, una revisión más exhaustiva sobre los mecanismos de comercialización del conocimiento, incluyendo a los intermediarios, y la accesibilidad por parte de los productores con menores recursos. Esto es crucial para garantizar un seguimiento más controlado y sostenible en el largo plazo, que vaya más allá de las metas específicas que se proponen las instituciones al formular cualquier programa o proyecto específico.

\section{Referencias}

Aguilar-Gallegos, N., Muñoz-Rodríguez, M., Santoyo-Cortés, H., Aguilar-Ávila, J., Klerkx, L. (2015). Information networks that generate economic value: A study on clusters of adopters of new or improved technologies and practices among oil palm growers in Mexico. Agricultural Systems, 135, pp. 122-132.

Arkesteijn, M., van Mierlo, B., Leeuwis, C. (2015). The need for reflexive evaluation approaches in development cooperation. Evaluation, 21 (1), pp. 99115.

Bennett, E. M., Cramer, W., Begossi, A., Cundill, G., Díaz, S., Egoh, B. N., \& Lebel, L. (2015). Linking biodiversity, ecosystem services, and human we11-being: three challenges for designing research for sustainability. Environmental Sustainability, 14, pp. 76-85.

Darnhofer, I., Schermer, M. W. Schneeberger (2008) Editorial: Continuity and change in organic farming - Philosophy, policy and practice. International Journal of Agricultural Resources, Governance and Ecology, 7, pp. 1-4.

Dentoni, D., Klerkx, L. (2015). Co-managing public research in Australian fisheries through convergence-divergence processes. Marine Policy, 60, pp. 259-271.

Dogliotti, S., García, M. C., Peluffo, S., Dieste, J. P., Pedemonte, A. J., Bacigalupe, G., Rossing, W. A. H. (2014). Co-innovation of family farm systems: A systems approach to sustainable agriculture. Agricultural Systems, 126, pp. 76-86.

Duckett, T., Pearson, S., Blackmore, S., Grieve, B. (2018). Agricultural Robotics: The Future of Robotic Agriculture. UK-RAS White papers. 
Engel, P., 1995. Facilitating innovation: an action-oriented approach and participatory methodology to improve innovative social practice in agriculture. PhD Thesis, Wageningen University, Wageningen, The Netherlands.

Fabricius, C., Cundill, G. (2014). Learning in adaptive management: insights from published practice. Ecology and Society, 19 (1).

FAO y Banco Mundial (2000). Agricultural Knowledge and Information Systems for Rural Development: Strategic Vision and Guiding Principles. Washington, DC: FAO.

Frisk, E., Larson, K. (2011). Educating for sustainability: Competencies \& practices for transformative action. Journal of Sustainability Education, 2(1), pp. 1-20.

Gerlak, A., Heikkila, T., Smolinski, S. L., Huitema, D., Armitage, D. (2018). Learning our way out of environmental policy problems: a review of the scholarship. Policy Sciences, 51 (3), pp. 335-371.

Godin B., Vinck D., 2017. Critical Studies of Innovation. Cheltenham, United Kingdom: Edward Elgar Publishing.

Hermans, F., Klerkx, L., Roep, D. (2015). Structural conditions for collaboration and learning in innovation networks: using an innovation system performance lens to analyse agricultural knowledge systems. The Journal of Agricultural Education and Extension, 21 (1), pp. 35-54.

INEC (2019). Encuesta Nacional de Hogares. San José, Costa Rica: INED.

Ingram, J., (2008). Agronomist-farmer knowledge encounters: an analysis of knowledge exchange in the context of best management practices in England, Agriculture and Human Values, 25, pp. 405-418.

Kilelu, C., Klerkx, L., Leeuwis, C. (2013). Unravelling the role of innovation platforms in supporting co-evolution of innovation: Contributions and tensions in a smallholder dairy development programme. Agricultural systems, 118, pp. 65-77.

Klerkx, L., Jansen, J. (2010). Building knowledge systems for sustainable agriculture: supporting private advisors to adequately address sustainable farm management in regular service contacts. Interna- tional Journal of Agricultural Sustainability, 8 (3), pp. 148-163.

Klerkx, L., Leeuwis, C. (2008). Balancing multiple interests: Embedding innovation intermediation in the agricultural knowledge infrastructure. Technovation, 28 (6), pp. 364-378.

Klerkx, L., Schut, M., Leeuwis, C., Kilelu, C. (2012). Advances in knowledge brokering in the agricultural sector: towards innovation system facilitation. ids Bulletin, 43 (5), pp. 53-60.

Klerkx, L., Van Mierlo, B., Leeuwis, C. (2012). Evolution of systems approaches to agricultural innovation: concepts, analysis and interventions. Farming Systems Research into the 21st century: The new dynamic, pp. 457-483.

Leeuwis, C. (2004). Communication for rural innovation: rethinking agricultural extension. New Jersey: John Wiley \& Sons.

Meléndez, J., Parker, B. (2018). Learning in participatory planning processes: Taking advantage of concepts and theories across disciplines. Planning Theory \& Practice, pp. 1-8.

Nagel, U. (1980) Institutionalisation of knowledge flows: an analysis of the extension role of two agricultural universities in India. Special issue of the Quarterly Journal of International Agriculture, 30, DLG Verlag, Frankfurt.

Petit S. (2015). Faut-il absolument innover? À la recherche d'une agriculture d'avant-garde. Courrier de l'environnement de l'Inra, 65, pp.19-28.

Röling, N. (1989) The Agricultural Research-technology Transfer Interface: A Knowledge Systems Perspective. The Hague: International Service for $\mathrm{Na}$ tional Agricultural Research (ISNAR).

Röling, N. (1992) The emergence of knowledge systems thinking: A changing perception of relationships among innovation, knowledge process and configuration. Knowledge and Policy: The International Journal of Knowledge Transfer and Utilization, 5, pp. 42-64.

Röling, N., Wagemakers, M. (Eds) (1998) Facilitating Sustainable Agriculture. Participatory Learning and Adaptive Management in Times of Environmental Uncertainty. Cambridge University Press: Cambridge. 
Schut, M., Rodenburg, J., Klerkx, L., van Ast, A., Bastiaans, L. (2014). Systems approaches to innovation in crop protection. A systematic literature review. Crop Protection, 56, pp. 98-108.

Schmid, J., Knierim, A., Knuth, U. (2016). Policy-induced innovations networks on climate change adaptation-An ex-post analysis of collaboration success and its influencing factors. Environmental Science \& Policy, 56, pp. 67-79.

SEPSA (2011). Política de estado para el sector agroalimentario y el desarrollo rural costarricense 20102021. San José, C.R.: MAG.

SEPSA (2014). Políticas para el sector agropecuario y el desarrollo de los territorios rurales 2015-2018. San José, C.R.: MAG.

SEPSA (2018). Lineamientos de política 2019-2022 para el Sector Agropecuario, Pesquero y Rural. Costa Rica: MAG.

Siebenhüner, B., Rodela, R., Ecker, F. (2016). Social learning research in ecological economics: A survey. Environmental Science \& Policy, 55, pp. 116-126.

Šūmane, S., Kunda, I., Knickel, K., Strauss, A., Tisenkopfs, T., des Ios Rios, I., Ashkenazy, A. (2018). Local and farmers' knowledge matters! How integrating informal and formal knowledge enhances sustainable and resilient agriculture. Journal of Rural Studies, 59, pp. 232-241.

Suškevičs, M., Hahn, T., Rodela, R., Macura, B., PahlWostl, C. (2018). Learning for social-ecological change: a qualitative review of outcomes across empirical literature in natural resource management. Journal of environmental planning and management, 61 (7), 1085-1112.

Temple, L., Sawadogo, E. (Eds.). (2018). Innovation processes in agro-ecological transitions in developing countries. John Wiley \& Sons.
Therond, O., Duru, M., Roger-Estrade, J., Richard, G. (2017). A new analytical framework of farming system and agriculture model diversities. A review. Agronomy for Sustainable Development, 37 (3), pp. 21.

Turner, J., Klerkx, L., White, T., Nelson, T., Everett-Hincks, J., Mackay, A., Botha, N. (2017). Unpacking systemic innovation capacity as strategic ambidexterity: How projects dynamically configure capabilities for agricultural innovation. Land Use Policy, 68, pp. 503-523.

Touzard, J. (2018). Agricultural and agrifood innovation in the 21st century: maintaining, erasing or reshaping its specificities? In Faure et a., (Ed.). Innovation and development in agricultural and food systems. France: Éditions Quæ, pp. 37-52.

Van Woerkum, C. (1990a) Het instrumentele nut van voorlichting in beleidsprocessen. Massacommunicatie, 18, pp. 263-78.

Wigboldus, S., Klerkx, L., Leeuwis, C., Schut, M., Muilerman, S., Jochemsen, H. (2016). Systemic perspectives on scaling agricultural innovations. A review. Agronomy for Sustainable Development, $36(3)$.

World Bank, 2012. Agricultural Innovation Systems - An Innovation Source Book. World Bank, Washington DC.

Yang, H., Klerkx, L., Leeuwis, C. (2014). Functions and limitations of farmer cooperatives as innovation intermediaries: Findings from China. Agricultural Systems, 127, pp. 115-125.

Yin, R. (1989). Case Study Research: Design and Methods, Applied social research Methods Series. Newbury Park, California: Sage 